\title{
Hamiltonian approach to relativistic star models
}

\author{
Kjell Rosquist! \\ Department of Physics, Stockholm University, Box 6730, 11385 Stockholm, Sweden \\ and \\ RGGR group, Chimie-Physique, CP231, Université Libre de Bruxelles, \\ Campus Plaine CP 231, 1050 Brussels, Belgium
}

\begin{abstract}
An ADM-like Hamiltonian approach is proposed for static spherically symmetric relativistic star configurations. For a given equation of state the entire information about the model can be encoded in a certain 2-dimensional minisuperspace geometry. We derive exact solutions which arise from symmetries corresponding to linear and quadratic geodesic invariants in minisuperspace by exploiting the relation to minisuperspace Killing tensors. A classification of exact solutions having the full number of integrations constants is given according to their minisuperspace symmetry properties. In particular it is shown that Schwarzschild's exterior solution and Buchdahl's $n=1$ polytrope solution correspond to minisuperspaces with a Killing vector symmetry while Schwarzschild's interior solution, Whittaker's solution and Buchdahl's $n=5$ polytrope solution correspond to minisuperspaces with a second rank Killing tensor. New solutions filling in empty slots in this classification scheme are also given. One of these new solutions has a physically reasonable equation of state and is a generalization of Buchdahl's $n=1$ polytrope model.
\end{abstract}

\footnotetext{
${ }^{1}$ E-mail: kr@physto.se
} 


\section{Introduction}

The subject of relativistic star configurations goes back to the very birth of general relativity itself when Schwarzschild gave his interior solution in 1916 [1]. Although a large body of literature now exists on the subject there still remain unsolved problems. For example, a theoretical understanding of some aspects of the interplay between the equation of state and the geometry of the model has only recently been achieved [2, 3. The stability of stellar models is another area in which only partial results are known concerning the relation between stability and the equation of state. In this paper we present a Hamiltonian formulation of the equations for spherically symmetric static equilibrium configurations. The Hamiltonian framework is then applied to show how known exact solutions are explained in terms of Killing tensor symmetries in a certain minisuperspace. A number of new exact solutions are also uncovered in this process.

Spherically symmetric gravitational fields belong to a class of models in which the spacetime is foliated or sliced by a family of homogeneous hypersurfaces. The causal character of the slicing is timelike. These facts lead one to compare and contrast these models with another class of models, namely the spatially homogeneous models, also hypersurface homogeneous but with a spacelike slicing. From a mathematical point of view the difference between star models and spatially homogeneous spacetimes is that the independent variable is a spacelike radial coordinate for stars while for spatially homogeneous models it is a timelike coordinate which is interpreted as a cosmic time function. The dramatic success and usefulness of Hamiltonian methods for exact solutions [ [ 1 , 5], solution structure [6], quantum cosmology [7] and visualization of the minisuperspace dynamics [8] make it natural to ask if the Hamiltonian methods might also be useful for star models. In fact the ADM formalism works also for timelike slicings of the spacetime [9]. In place of the lapse function one must then introduce a radial gauge function. One consequence of this is that the Schwarzschild coordinates are unsuitable as a starting point for the Hamiltonian formulation. Instead they will correspond to a certain choice of momentum dependent radial gauge function. Also, as explained below one must be careful when introducing the matter terms to obtain a correct Hamiltonian. This paper is intended to serve two main purposes the first of which is to outline the Hamiltonian approach to star models in general while the second main purpose is to classify those exact solutions which are related to symmetries of the relativistic equations for static equilibrium.

The importance of the Hamiltonian approach for exact solutions stems from the possibility to define a dynamical minisuperspace geometry which carries all information about the dynamics. We shall define such a dynamical geometry by using a special radial gauge function to be referred to as the Jacobi gauge. This is analogous to the Jacobi lapse which was introduced for spatially homogenous models in 110, 11. The resulting Jacobi geometry is conformally related to minisuperspace geometries corresponding to other choices of lapse function. Given such a dynamical geometry the problem of finding and classifying exact solutions becomes a matter of studying geodesic orbits, a subject which has been treated from many points of view in the literature. There are two different mechanisms by which exact solutions can occur. A system of differential equations can be reduced either by means of a symmetry or by means of an invariant submanifold leading to symmetry solutions and submanifold solutions, corresponding to the classical notions of general and particular solutions respectively. Broadly speaking, symmetry solutions are more useful since they allow one to impose arbitrary initial conditions. Fortunately, they are also more amenable to systematic study by using the theory of Lie symmetries for differential equations.

In the context of relativistic star models initial conditions correspond to matching conditions at the surface of the star and possibly inside the star to match the solution to one with a different equation of state above a certain pressure. For spherically symmetric models the matching condition is simply that the pressures of the two solutions must be equal at the boundary. In particular, the surface of the star is defined as the point of zero pressure and this automatically matches the solution to the Schwarzschild vacuum solution by Birkhoff's theorem [9]. In addition, a solution which goes all the way to the center of the star must be regular there. This is the well-known condition of elementary flatness. It is discussed in section 2 in the context of the Hamiltonian framework. An alternative to using symmetry solutions is to assume a certain functional form for the radial dependence of a metric component (e.g. [12]) or for the energy density (e.g. [13]). If the functional form involves some arbitrary parameters, then one may obtain physically reasonable submanifold solutions by adjusting those parameters in order to satisfy the boundary conditions.

In the geometric formulation of the dynamics ${ }^{2}$ the Hamiltonian takes the purely kinetic form $H_{J}=\frac{1}{2} J^{A B} p_{A} p_{B}$ where the Jacobi metric $J_{A B}$ is the metric of minisuperspace in the Jacobi gauge. In this picture the star configurations correspond to the timelike geodesics of the Jacobi geometry. The simplest symmetries of $H_{J}$ are those which are related to Killing vectors of $J_{A B}$. It is well-known that a Killing vector $\xi^{A}$ gives rise to a linear constant of the motion

${ }^{2}$ The word dynamics is somewhat inappropriate here since we are dealing with static models. However, the radial variable will be seen to play a role which is identical from the mathematical point of view to the role played by the time in the closely related spatially homogeneous models. In particular the radial metric coordinate serves as the independent variable for the Hamiltonian. Lacking a more suitable term we will refer to the Hamiltonian equations of star configurations as "dynamics". 
for $H_{J}$ given by $\xi^{A} p_{A}$. Similarly, constants of the motion may exist which are homogeneous polynomials of quadratic or higher degree in the momenta. In the quadratic case such constants of the motion have the form $\xi^{A B} p_{A} p_{B}$ where $\xi^{A B}$ are the components of a geometric object known as a second rank Killing tensor [14]. In general, a constant of the motion of the $n$ 'th degree corresponds to a $n$ 'th rank Killing tensor. In particular a Killing vector is a Killing tensor of rank one. The precise relation between Killing tensors and Lie symmetries of the geodesic equations was given in [15].

In the ADM formulation the static star configurations are 1+1-dimensional Hamiltonian systems. A criterion for the existence of Killing vectors and second rank Killing tensors for such systems was given in [5]. It was also shown in [e] how a Killing vector or a Killing tensor can be used to obtain variables which are adapted to the symmetry. Such symmetry adapted variables are needed for explicit integration of the equations of motion. There is a large number of exact solutions in the literature (see [14] for a partial list) some of them symmetry solutions and some submanifold solutions. One of our main objectives is to identify the symmetry solutions.

We shall use the Killing tensor criterion of [5] to look for integrable relativistic star models. While a general solution to this problem is not available a present, even for Killing tensors up to second rank, we will be able to make substantial progress by using a certain ansatz for the symmetry adapted variables. The resulting family of solutions includes all (to this author's knowledge) known symmetry solutions as well as some new solutions. There is no guarantee that the symmetry solutions are physically reasonable. We shall therefore use the following rough criterion to separate solutions with a more reasonable equation of state from the obviously unphysical solutions. We consider the equation of state to be physical if the conditions $p \geq 0, \rho \geq 0$ and $0 \leq \mathrm{d} p / \mathrm{d} \rho \leq 1$ are satisfied. The last of these conditions ensures that the velocity of sound is well-defined by $v_{\text {sound }}=\sqrt{\mathrm{d} p / \mathrm{d} \rho}$ and that it does not exceed the speed of light. Note that an equation of state which is defined by some function $p=f(\rho)$ may be physical at some densities but unphysical at others. Our condition is in fact rather strict and excludes for example the incompressible fluid used in Schwarzschild's interior solution. One of the new solutions found in this work is physical according to our criterion and generalizes Buchdahl's $n=1$ polytrope solution [16].

\section{Hamiltonian formulation of static models}

We consider static spherically symmetric matter configurations where the matter is a perfect fluid described by some equation of state $p=f(\rho)$. The stress-energy tensor is given by $T_{\alpha \beta}=(\rho+p) u_{\alpha} u_{\beta}+p g_{\alpha \beta}$ and the metric is required to satisfy the Einstein equations $G_{\alpha \beta}=\kappa T_{\alpha \beta}$. The metric of spherically symmetric models is usually written as 9

$$
d s^{2}=-e^{2 \nu} d t^{2}+e^{2 \lambda} d r^{2}+r^{2} \mathrm{~d} \Omega^{2},
$$

where

$$
\mathrm{d} \Omega^{2}=\mathrm{d} \theta^{2}+\sin ^{2} \theta \mathrm{d} \phi^{2},
$$

is the metric of the 2-sphere. One reason why this form has been preferred in the past is that the Schwarzschild $r$ variable is invariantly defined by its relation $A=4 \pi r^{2}$ to the area $A$ of a 2 -surface given by $d t=d r=0$. One lesson to be learned, though, from the experience of spatially homogeneous models is that no preferred set of variables exists which is suitable for all situations. In particular, for the Hamiltonian method we need to modify the form (化) of the metric. Using the analogy with spatially homogeneous models a natural starting point for a Hamiltonian formulation is to write the metric in the form

$$
d s^{2}=-e^{2 \beta^{3}} d t^{2}+N^{2} d R^{2}+e^{2 \beta^{1}} \mathrm{~d} \Omega^{2},
$$

where $N$ is a radial gauge function analogous to the lapse function in formulations with a spacelike slicing. For static models $N$ is a function of the radial coordinate $R$. The relation to conventional variables is given be the relations

$$
\beta^{3}=\nu, \quad e^{\beta^{1}}=r, \quad N d R=e^{\lambda} d r .
$$

Therefore the conventional variables correspond to the specific choice of radial gauge, $N_{\mathrm{S}}$, for which the radial variable is precisely the Schwarzschild $r$ variable. We shall refer to this choice as the Schwarzschild radial gauge. In the terminology of $\sqrt{17}$ this is an example of an intrinsic gauge choice. The square of the Schwarzschild gauge function, $N_{\mathrm{S}}{ }^{2}$, coincides with the "Schwarzschild correction factor" of Harrison et al. [18] This factor determines whether or not there is a conical singularity at the center of the star model. It can be computed in an arbitrary radial gauge by the relation (a comma will denote differentiation throughout the paper)

$$
N_{\mathrm{S}}=e^{\lambda}=N\left(r_{, R}\right)^{-1}=N e^{-\beta^{1}}\left(\beta_{, R}^{1}\right)^{-1} .
$$


As for spacelike slicings the Misner variables $\beta^{A}$ are defined by

$$
\begin{aligned}
\beta^{0}=\frac{1}{3}\left(2 \beta^{1}+\beta^{3}\right), & \beta^{1}=\beta^{0}+\beta^{+}, \\
\beta^{+}=\frac{1}{3}\left(\beta^{1}-\beta^{3}\right), \beta^{3} & =\beta^{0}-2 \beta^{+} .
\end{aligned}
$$

diagonalize the kinetic energy in the ADM Hamiltonian. It is also convenient to define the Taub radial gauge by $N=N_{T}=12 e^{3 \beta^{0}}$ 19]. Ising the Lorentz frame $\omega^{0}=e^{\beta^{3}} d t, \omega^{1}=N_{T} d R, \omega^{2}=e^{\beta^{1}} \mathrm{~d} \theta, \omega^{3}=e^{\beta^{1}} \sin \theta \mathrm{d} \phi$ the Einstein equations for the metric (3) become

$$
\begin{aligned}
& G_{00}=-\frac{1}{24} e^{-6 \beta^{0}} T-\frac{1}{72} e^{-6 \beta^{0}}\left(\beta_{, R R}^{0}+\beta_{, R R}^{+}\right)+e^{-2\left(\beta^{0}+\beta^{+}\right)}=\kappa \rho, \\
& G_{11}=-\frac{1}{24} e^{-6 \beta^{0}} T-e^{-2\left(\beta^{0}+\beta^{+}\right)}=\kappa p \\
& G_{22}=G_{33}=\frac{1}{24} e^{-6 \beta^{0}} T+\frac{1}{144} e^{-6 \beta^{0}}\left(2 \beta_{, R R}^{0}-\beta_{, R R}^{+}\right)=\kappa p
\end{aligned}
$$

where

$$
T=\frac{1}{2}\left[-\left(\beta_{, R}^{0}\right)^{2}+\left(\beta_{, R}^{+}\right)^{2}\right] .
$$

Note that the above equations are given in the Taub radial gauge. Eliminating $T$ and solving for the second derivatives yields

$$
\begin{aligned}
& \beta_{, R R}^{0}=96 e^{4 \beta^{0}-2 \beta^{+}}+24 \kappa e^{6 \beta^{0}}(5 p-\rho), \\
& \beta_{, R R}^{+}=48 e^{4 \beta^{0}-2 \beta^{+}}-48 \kappa e^{6 \beta^{0}}(p+\rho) .
\end{aligned}
$$

The ADM Hamiltonian for a spherically symmetric static fluid should be given by 9,17

$$
H=-2 N^{(3)} V n^{\alpha} n^{\beta}\left(G_{\alpha \beta}-\kappa T_{\alpha \beta}\right),
$$

where ${ }^{(3)} V=e^{3 \beta^{0}}$ is the 3 -volume element (as usual only defined only up to a constant factor) and $n^{\alpha}$ is the unit normal to the homogeneous hypersurfaces. It is constrained by the Einstein equations to the zero energy surface, $H=0$. Using the prescription (10) the Hamiltonian takes the form

$$
H=\frac{1}{2} \mathcal{N}\left(-p_{0}{ }^{2}+p_{+}{ }^{2}\right)+24 \mathcal{N} e^{4 \beta^{0}-2 \beta^{+}}+24 \kappa \mathcal{N} e^{6 \beta^{0}} p\left(\beta^{0}, \beta^{+}\right),
$$

where $\mathcal{N}$ is a relative radial gauge function defined by $\mathcal{N}=N / N_{T}$ and $p$ is the pressure written as a function of the metric variables. As discussed above the Hamiltonian is constrained by the Einstein equations to the zero energy surface, $H=0$.

At this point it is not yet clear how the variation of the pressure function $p\left(\beta^{0}, \beta^{+}\right)$is to be done in the Hamiltonian framework. In fact it turns out that the pressure cannot be varied independently with respect to $\beta^{0}$ and $\beta^{+}$. This can be understood if we write down the field equations obtained from the Hamiltonian (11) and compare them with the Einstein equations. For that purpose it is convenient to use the corresponding Lagrangian in the Taub radial gauge $\mathcal{N}=1$

$$
L=\frac{1}{2}\left[-\left(\beta_{, R}^{0}\right)^{2}+\left(\beta_{, R}^{+}\right)^{2}\right]-24 e^{4 \beta^{0}-2 \beta^{+}}-24 \kappa e^{6 \beta^{0}} p\left(\beta^{0}, \beta^{+}\right) .
$$

The equations of motion of this Lagrangian are

$$
\begin{array}{r}
\frac{\partial L}{\partial \beta^{0}}-\frac{\mathrm{d}}{\mathrm{d} t} \frac{\partial L}{\partial \beta_{, R}^{0}}=-96 e^{4 \beta^{0}-2 \beta^{+}}-144 \kappa e^{6 \beta^{0}} p-24 \kappa e^{6 \beta^{0}} \frac{\partial p}{\partial \beta^{0}}+\beta_{, R R}^{0}=0 \\
\frac{\partial L}{\partial \beta^{+}}-\frac{\mathrm{d}}{\mathrm{d} t} \frac{\partial L}{\partial \beta_{, R}^{+}}=48 e^{4 \beta^{0}-2 \beta^{+}}-24 \kappa e^{6 \beta^{0}} \frac{\partial p}{\partial \beta^{+}}-\beta_{, R R}^{+}=0 .
\end{array}
$$

For these equations to coincide with the Einstein equations (9) we see by inspection that the pressure function $p\left(\beta^{0}, \beta^{+}\right)$must satisfy the relations

$$
\frac{\partial p}{\partial \beta^{0}}=-(p+\rho), \quad \frac{\partial p}{\partial \beta^{+}}=2(p+\rho),
$$

\footnotetext{
${ }^{3}$ We use capital indices $(A, B, \ldots)$ for the Misner variables taking the values $0,+$. (The third Misner variable $\beta^{-}$is not needed in this context.)

${ }^{4}$ This gauge was originally introduced by Taub for spatially homogeneous models. Misner called it the supertime gauge in that context.

${ }^{5}$ In the context of spacelike slicings Jantzen [20] uses the notation $x=\mathcal{N}^{-1}$ while Ashtekar [21] uses the term densitized lapse for $\stackrel{N}{\sim}=12 \mathcal{N}$.
} 
leading to

$$
2 \frac{\partial p}{\partial \beta^{0}}+\frac{\partial p}{\partial \beta^{+}}=0
$$

This equation shows that $p$ must be considered as a function of $\nu=\beta^{3}=\beta^{0}-2 \beta^{+}$in order that Hamilton's equations as derived from the Hamiltonian (11) represent the Einstein equations correctly. We have thus shown that the Hamiltonian for spherically symmetric static models is given by

$$
H=\frac{1}{2} \mathcal{N}\left(-p_{0}{ }^{2}+p_{+}{ }^{2}\right)+24 \mathcal{N} e^{4 \beta^{0}-2 \beta^{+}}+24 \kappa \mathcal{N} e^{6 \beta^{0}} \mathcal{P}(\nu)
$$

where we have introduced the notation $\mathcal{P}(\nu)=p$ for the pressure function. The momenta can be expressed in terms of the velocities by

$$
\begin{gathered}
p_{0}=-\mathcal{N}^{-1} \beta_{, R}^{0}, \\
p_{+}=\mathcal{N}^{-1} \beta_{, R}^{+} .
\end{gathered}
$$

A number of remarks about the nature of the Hamiltonian (16) are in order. Interestingly, it is the pressure rather than the energy density which appears in the matter term by contrast to the Hamiltonians of models with spacelike slicings such as minisuperspace models for example. Since the kinetic metric carries a Lorentzian signature we can characterize variables as being timelike, spacelike or null with respect to that metric. In particular $\beta^{1}=\log r$ is a null variable which increases with distance from the center of the star. It follows that any timelike or null variable must also be increasing functions. Another useful feature of the Hamiltonian (16) is the freedom to perform Lorentz transformations which preserve the kinetic energy. For some purposes we will also use null variables defined by

$$
\begin{aligned}
w & =\beta^{0}+\beta^{+}=\beta^{1}, \\
v & =\beta^{0}-\beta^{+}=\frac{1}{3}\left(\beta^{1}+2 \beta^{3}\right), \quad \nu=\beta^{3}=\frac{1}{2}(-w+3 v) .
\end{aligned}
$$

The pressure is related to the energy density by the conservation equation

$$
-\frac{\mathrm{d} p}{\mathrm{~d} \nu}=\rho+p
$$

This is consistent with the variational constraint (15) on the pressure. Therefore the natural procedure to integrate the system is to first specify the equation of state $p=p(\rho)$, then use the conservation equation to write $p$ as a function of $\nu$ and then insert that function into the Hamiltonian. The resulting Hamilton's equations should then be integrated with suitable boundary conditions. We shall require as usual that the metric is well defined at the center so that it satisfies the condition of elementary flatness there. In addition it is necessary that the pressure is zero at the surface of the star in order that the geometry can be joined smoothly to the Schwarzschild metric in the exterior. To see how to express the elementary flatness condition analytically in terms of our variables we write the spatial 3 -metric as

$$
N_{\mathrm{S}}^{2} \mathrm{~d} r^{2}+r^{2} \mathrm{~d} \Omega^{2}
$$

Elementary flatness at the center requires that the Schwarzschild radial gauge function $N_{\mathrm{S}}$ (expressed in a general gauge in equation (5)) tends to unity [18. Using null variables the condition becomes

$$
N_{\mathrm{S}}^{-1}=N^{-1} e^{w} \frac{\mathrm{d} w}{\mathrm{~d} R} \rightarrow 1
$$

To analyze how the zero pressure limit appears in configuration space let $\rho_{s}$ be the value of $\rho$ at the surface. It is useful to distinguish between the cases $\rho_{s} \neq 0$ (liquid-like equation of state) and $\rho_{s}=0$ (gas-like equation of state). In the first case when the pressure tends to zero it follows from (19) that $\nu$ must tend to a finite constant, $\nu_{s}$. For a given equation of state and star mass $m$, let $\mathcal{Q}_{\mathrm{s}}(m)$ be the point in configuration space where $p=0$. By varying $m$, the points $\mathcal{Q}_{\mathrm{s}}(m)$ form a curve in configuration space representing the star surface for the given equation of state. Since $p$ is a function of the spacelike variable $\nu$ it follows that the star surface curve is a timelike plane given by $\nu=\nu_{s}$. In the case $\rho_{s}=0$ one cannot use (19) to draw the same conclusions.

The center of the star can be defined as the limit of zero 2 -volume, $e^{2 \beta^{1}} \rightarrow 0$ or equivalently $w=\beta^{0}+\beta^{+} \rightarrow-\infty$. Thus, this limit is lightlike in configuration space. Just as for minisuperspace models it is possible to perform a Penrose compactification scheme for the configuration space (cf. [8]). By the above remarks it then follows that the star center would be located at $\Im_{-}$or $i_{-}$. A more refined argument can be made to show that the center of the star is actually located at $i_{-}$but we will not pursue this point further in this paper.

A global quantity of particular interest is the mass function $m(R)$ given by

$$
m(R)=4 \pi \int_{0}^{R} \rho R^{\prime 2} d R^{\prime}=4 \pi \int_{-\infty}^{\beta^{1}} \rho e^{3 \beta^{\prime 1}} \mathrm{~d} \beta^{\prime 1} .
$$


In particular the total mass of the star is the value of the mass function at the surface, $M=m\left(R_{\mathrm{s}}\right)$. The total mass can also be computed without integration by using the matching conditions at the surface for one of the two metric components which depend on the mass. To see how this works for the component $e^{2 \beta^{3}}=e^{2 \nu}$ we use the notation $Z=e^{2 \nu}$ and note that for the Schwarzschild exterior geometry we have $Z=1-2 M / r$. However, we cannot use this relation as it stands to calculate $M$ because the value $Z_{\mathrm{s}}$ is not invariantly defined since we can always rescale the time variable by a constant factor. Taking that gauge freedom into account we have

$$
Z=B(1-2 M / r)
$$

where $B$ is some arbitrary constant. To eliminate the gauge factor $B$ we differentiate (23) with respect to $R$ and then solve for $M$. Evaluating the resulting expression at the surface yields

$$
M=\frac{1}{2}\left[r\left(1+\frac{Z}{r Z_{, r}}\right)^{-1}\right]_{\mathrm{s}}=\frac{1}{2}\left[r\left(1+\frac{r_{, R} Z}{r Z_{, R}}\right)^{-1}\right]_{\mathrm{s}},
$$

for Schwarzschild and arbitrary radial gauges respectively. For the second way of calculating the mass we make use of the metric coefficient $N_{\mathrm{S}}^{2}=e^{2 \lambda}=(1-2 M / r)^{-1}$ the last equality holding for the external geometry. The value of this coefficient is invariant since the Schwarzschild radial coordinate itself is invariantly defined. Solving for the mass gives

$$
M=\frac{1}{2}\left[r\left(1-N_{\mathrm{S}}{ }^{-2}\right)\right]_{\mathrm{s}} .
$$

Which of the expressions for the mass one uses is a matter of convenience. We note in passing that the two expressions together imply the identity

$$
\left(N_{\mathrm{S}}^{2}\right)_{\mathrm{s}}=1+\left(\frac{r Z, R}{r_{, R} Z}\right)_{\mathrm{s}} .
$$

It is sometimes useful to adapt the system to the $\beta^{3}$ variable. This is achieved by performing a boost with velocity $1 / 2$

$$
\begin{aligned}
& \beta^{0}=\frac{1}{\sqrt{3}}\left(2 \bar{\beta}^{0}+\bar{\beta}^{+}\right), \\
& \beta^{+}=\frac{1}{\sqrt{3}}\left(\bar{\beta}^{0}+2 \bar{\beta}^{+}\right), \quad \beta^{3}=\nu=-\sqrt{3} \bar{\beta}^{+},
\end{aligned}
$$

leading to the Hamiltonian

$$
H=\frac{1}{2} \mathcal{N}\left(-\bar{p}_{0}{ }^{2}+\bar{p}_{+}{ }^{2}\right)+24 \mathcal{N} e^{2 \sqrt{3} \bar{\beta}^{0}}+24 \mathcal{N} \kappa e^{4 \sqrt{3} \bar{\beta}^{0}} e^{2 \sqrt{3} \bar{\beta}^{+}} \mathcal{P}\left(-\sqrt{3} \bar{\beta}^{+}\right) .
$$

Note that this mathematical structure is similar to that of a negative curvature Robertson-Walker model minimally coupled to a scalar field (see [17]). The only difference is in the values of the coefficients of $\bar{\beta}^{0}$. We shall also need the Hamiltonian in the null variables (18)

$$
H=-2 \mathcal{N} p_{w} p_{v}+24 \mathcal{N} e^{w+3 v}+24 \kappa \mathcal{N} e^{3(w+v)} \mathcal{P}(\nu)
$$

recalling that $\nu$ is given by (18). When integrating the solutions it is useful to write the Hamiltonian in terms of the velocities

$$
H=-\frac{1}{2} \mathcal{N}^{-1} w_{, R} v_{, R}+24 \mathcal{N} e^{w+3 v}+24 \kappa \mathcal{N} e^{3(w+v)} \mathcal{P}(\nu) .
$$

Exact solutions can be either symmetry solutions with the full number of integration constants or submanifold solutions (also called special or particular solutions) which arises whenever an invariant submanifold can be expressed as an explicit functional relation on the phase space. Since the latter solutions are only particular solutions they cannot in general be made to satisfy the physical boundary conditions. For the models we are considering in this paper the symmetry solutions are also general solutions since a single symmetry is always sufficient to integrate a 2-dimensional Hamiltonian system.

The analysis of the symmetries of finite-dimensional Hamiltonians constrained to a zero energy surface

$$
H(q, p)=T(q, p)+U(q)=0
$$

with a quadratic kinetic energy function $T(q, p)=\frac{1}{2} h^{A B}(q) p_{A} p_{B}$ is much facilitated by going to the Jacobi gauge $\mathcal{N}=\mathcal{N}_{J}=|2 U|^{-1}$ in which the problem is reduced to finding Killing vectors and Killing tensors in the Jacobi geometry

$$
\mathrm{d} s_{J}^{2}=2|U| h_{A B} \mathrm{~d} q^{A} \mathrm{~d} q^{B} .
$$

The method has been described in a series of papers dealing with spatially homogeneous models (see [4, 月, 17] and references therein). 
The Jacobi metric for the static spherically symmetric fluid can be read off from (29) with the result (after rescaling by a numerical conformal factor)

$$
\mathrm{d} s_{J}^{2}=-2 G \mathrm{~d} w \mathrm{~d} v, \quad G=e^{w+3 v}+\kappa e^{3(w+v)} \mathcal{P}(\nu) .
$$

Any Killing tensor of this metric gives rise to a constant of the motion and a corresponding Lie symmetry of the Einstein equations. Whether or not such a symmetry exists depends on the form of the pressure function $\mathcal{P}(\nu)$. In the next section we will see how such functions can be found.

\section{$3 \quad$ Killing tensor conditions and pressure functions}

In this section we introduce an ansatz which makes it possible to determine equations of state for which the Jacobi metric (33) admits a second rank Killing tensor. This will include Killing vector cases as well. Since we are using an ansatz we do not expect to find all possible solutions but it turns out, however, that we do recover a substantial fraction of the solutions which have been discussed in the literature for their physical or mathematical interest. Moreover, we show elsewhere 22 that the ansatz actually gives all possible Killing vector solutions .

The Jacobi metric is $(1+1)$-dimensional and therefore admits three distinct types of Killing tensors as shown in [5]. The Killing tensor $K_{A B}$ may have either two non-null eigenvectors or a single null eigenvector. When there are non-null eigenvectors two subcases arise, one which corresponds to standard Hamilton-Jacobi separability and another one for which the equations of motion can be decoupled in suitable complex variables. The possibility of non-Hamilton-Jacobi separation is a consequence of the indefinite nature of the Jacobi metric.

We now state the necessary and sufficient conditions for the existence of a second rank Killing tensor in the Jacobi geometry (33). A set of null coordinates $(W, V)$ are adapted to a second rank Killing tensor of the 2-dimensional Lorentzian metric

$$
d s^{2}=-2 G(W, V) \mathrm{d} W \mathrm{~d} V,
$$

precisely if one of the following conditions hold [5]:

$$
\begin{array}{rrr}
G_{, W W}=0 \text { or } G_{V V} & =0, & \text { null Killing tensor, linear decoupling, } \\
G_{, W W}-G_{, V V} & =0, & \text { non-null Killing tensor, Hamilton-Jacobi separation, } \\
G_{, W W}+G_{, V V} & =0, & \text { non-null Killing tensor, complex decoupling. }
\end{array}
$$

The Killing vector case is contained in the Hamilton-Jacobi separation case. As a first step we check if the original null coordinates $(w, v)$ defined in (18), modulo a possible scaling, can be made symmetry adapted in this sense by some appropriate choice of pressure function $\mathcal{P}(\nu)$. To use the above conditions (35) we define null variables $(W, V)$ by $w=k W, v=V$. This corresponds to a boost combined with a trivial scaling of the original Misner variables $\beta^{A}$. The metric function can then be written as

$$
G=e^{k W+3 V}+\kappa e^{3 k W+3 V} \mathcal{P}(\nu)
$$

Calculating the second derivatives and representing them in the variables $(\nu, V)$, they can be displayed in the form

$$
\begin{aligned}
e^{-12 V+6 \nu} G_{, W W} & =k^{2} e^{-6 V}+\kappa k^{2}\left[9 \mathcal{P}(\nu)-3 \mathcal{P}^{\prime}(\nu)+\frac{1}{4} \mathcal{P}^{\prime \prime}(\nu)\right], \\
e^{-12 V+6 \nu} G_{, V V} & =9 e^{-6 V}+9 \kappa\left[\mathcal{P}(\nu)+\mathcal{P}^{\prime}(\nu)+\frac{1}{4} \mathcal{P}^{\prime \prime}(\nu)\right] .
\end{aligned}
$$

It is apparent from these formulas that $G_{, W W}$ and $G_{, V V}$ cannot separately be set to zero. It follows that there is no null Killing tensor adapted to these coordinates. Complex decoupling is also impossible since the coefficient of the first term of the right hand side has the same sign in both expressions in (37). Consequently that term cannot be canceled when taking the sum. We can, however, obtain Hamilton-Jacobi separation by taking the difference

$$
\begin{array}{r}
e^{-12 V+6 \nu}(G, W W-G, V V)=\left(k^{2}-9\right) e^{-6 V} \\
+\kappa\left[9\left(k^{2}-1\right) \mathcal{P}(\nu)-3\left(k^{2}+3\right) \mathcal{P}^{\prime}(\nu)+\frac{1}{4}\left(k^{2}-9\right) \mathcal{P}^{\prime \prime}(\nu)\right] .
\end{array}
$$

It follows that the right hand side vanishes identically precisely when $k^{2}=9$ and $\mathcal{P}^{\prime}(\nu)=2 \mathcal{P}(\nu)$. Comparison with equation (19) then leads to the equation of state $\rho+3 p=0$. However, this is only a special case of the more general equation of state $\rho+3 p=$ constant for which the general exact solution was found by Whittaker [23] and which is shown to correspond to a null Killing tensor in section 4 .

Let us now consider more general transformations than the simple null scaling used above. To help us in the search for suitable conformal transformations we examine the structure of the matter potential for some equations of state of physical interest. For spatially homogeneous cosmological models with the usual equation of state $p=(\gamma-1) \rho$ 
Table 1: Expressions for pressure functions $p=\mathcal{P}(\nu)$ for some equations of state where $\rho_{0}, p_{0}, p_{*}$ and $p_{\star}$ are constants. The corresponding fluid potentials are sums of exponentials for integer $n$. The expressions may be subjected to a gauge translation of the metric variable $\nu=\beta^{3}$. The type codes stand for polytrope-gamma law (PG), Tooper's equation of state (T), relativistic polytrope (RP), incompressible fluid-gamma law (IG), incompressible fluid (I), gamma law (G) and Buchdahl's $n=1$ and $n=5$ polytropes (BP1 and BP5). The velocity of sound for Buchdahl's $n=1$ polytrope is less than the speed of light for pressures $p<p_{*}$. Buchdahl's $n=5$ polytrope satisfies $p<\rho$ for $p<p_{\star}$.

\begin{tabular}{lll}
\hline$p=\mathcal{P}(\nu)$ & equation of state & type \\
\hline$(B+1)^{-n-1} A^{n+1}\left[e^{-(B+1) \nu /(n+1)}-1\right]^{n+1}$ & $\rho=A p^{n /(n+1)}+B p$ & $\mathrm{PG}$ \\
$(n+1)^{-n-1} K^{-n}\left(e^{-\nu}-1\right)^{n+1}$ & $\rho=(p / K)^{n /(n+1)}+n p$ & $\mathrm{~T}$ \\
$K^{-n}\left[e^{-\nu /(n+1)}-1\right]^{n+1}$ & $p=K \rho^{1+1 / n}$ & $\mathrm{RP}$ \\
$(n+1)^{-1} \rho_{0}\left[e^{-(n+1) \nu}-1\right]$ & $\rho=\rho_{0}+n p,[n=1 /(\gamma-1)]$ & $\mathrm{IG}$ \\
$\rho_{0}\left(e^{-\nu}-1\right)$ & $\rho=\rho_{0}$ & $\mathrm{I}$ \\
$p_{0} e^{-(n+1) \nu}$ & $p=(\gamma-1) \rho,[n=1 /(\gamma-1)]$ & $\mathrm{G}$ \\
$9 p_{*}\left(e^{2 \nu}-1\right)^{2}$ & $\rho=12 \sqrt{p_{*} p}-5 p$ & $\mathrm{BP} 1$ \\
$6^{-6} 7^{5} p_{\star} e^{-\nu}\left(1-e^{\nu}\right)^{6}$ & $p=\rho^{6 / 5} /\left(7 p_{\star}^{1 / 5}-6 \rho^{1 / 5}\right)$ & $\mathrm{BP} 5$ \\
\hline
\end{tabular}

the minisuperspace potential is a sum of terms which are exponentials of linear combinations of the Misner variables. Integrating the conservation equation (19) one finds that this is also true for many of the equations of state which have been discussed in connection with relativistic star models. Most such equations of state are special cases of the relation

$$
\rho=A p^{n /(n+1)}+B p,
$$

which interpolates between a relativistic polytrope $p=K \rho^{1+1 / n}$ of index $n$ for small pressures and a gamma-law equation of state $p=(\gamma-1) \rho$ for large pressures. The choice $A=K^{-n /(n+1)}, B=n$ gives the relativistic ideal gas considered by Tooper 24. Other special cases of (39) are $\rho=\rho_{0}+(\gamma-1)^{-1} p$ and an incompressible fluid $\rho=\rho_{0}$. To this list can be added Buchdahl's $n=1$ polytrope while Buchdahl's $n=5$ polytrope is an example of an equation of state which is not of the form (39). The exponential representations of the above mentioned equations of state are given in Table 1. Symmetry adapted variables for solvable spatially homogeneous models are either linear combinations of the Misner variables themselves or exponentials of null Misner variables. Also, as noted in [17 the null variables $(W, V)$ defined by $W=e^{w}, V^{3 / 2}=e^{v}$ are symmetry adapted for the Schwarzschild interior solution. In all cases the symmetry adapted potential turns out to be a polynomial in the symmetry adapted variables. These observations together lead us to the ansatz

$$
W^{\alpha}=e^{w}=r, \quad V^{\beta}=e^{v},
$$

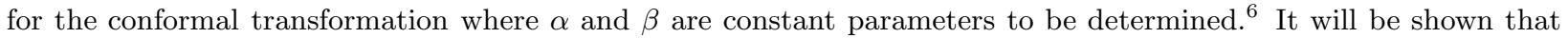
the above ansatz for the conformal transformation can be used to recover all (to this author's knowledge) known equations of state which lead to a general solution of the equations of motion. In addition the ansatz also leads some new exact solutions.

In the null variables defined by the ansatz (40) the Jacobi metric (33) takes the form (upon rescaling by a constant factor)

$$
\begin{aligned}
d s_{J}{ }^{2} & =-2 G \mathrm{~d} W \mathrm{~d} V, \\
G & =W^{\alpha-1} V^{3 \beta-1}+\kappa W^{3 \alpha-1} V^{3 \beta-1} f(Z),
\end{aligned}
$$

and we have defined $Z=e^{2 \beta^{3}}=W^{-\alpha} V^{3 \beta}$ and $f(Z)=p=\mathcal{P}\left(\frac{1}{2} \log Z\right)$. Next we ask under what conditions on the parameters $\alpha$ and $\beta$ and on the function $f(Z)$ do the variables $W$ and $V$ become symmetry adapted with respect to a Killing vector or a second rank Killing tensor. For this purpose we use the conditions (35) and we therefore compute the second derivatives of the metric conformal factor from (41) with the result

$$
\begin{array}{r}
G, W W=(\alpha-1)(\alpha-2) W^{\alpha-3} V^{3 \beta-1}+(3 \alpha-1)(3 \alpha-2) W^{3 \alpha-3} V^{3 \beta-1} f(Z) \\
-\alpha(5 \alpha-3) W^{2 \alpha-3} V^{6 \beta-1} f^{\prime}(Z)+\alpha^{2} W^{\alpha-3} V^{9 \beta-1} f^{\prime \prime}(Z) \\
G_{, V V}=(3 \beta-1)(3 \beta-2) W^{\alpha-1} V^{3 \beta-3}+(3 \beta-1)(3 \beta-2) W^{3 \alpha-1} V^{3 \beta-3} f(Z) \\
+9 \beta(3 \beta-1) W^{2 \alpha-1} V^{6 \beta-3} f^{\prime}(Z)+9 \beta^{2} W^{\alpha-1} V^{9 \beta-3} f^{\prime \prime}(Z) .
\end{array}
$$

\footnotetext{
${ }^{6}$ One could also use the slightly more general transformation obtained by replacing the first relation by the scaled form $e^{w}=(k W)^{\alpha}$. However, it turns out that this additional freedom does not lead to any new solvable models.
} 


\section{$4 \quad$ Null Killing tensor solutions}

In this section we determine the equations of state for which there exists a null Killing tensor in the minisuperspace Jacobi geometry. From the conditions $(i)-(i i i)$ in section 3 we know that the Jacobi metric (33) admits a null Killing tensor if one of $G, W W$ or $G, V V$ vanishes identically. Our aim is to find all possible functions $f$ for which this is the case subject to the ansatz (40). To facilitate this analysis we replace the variable set $(W, V)$ in $(42)$ by $(Z, V)$ with the result

$$
\begin{gathered}
G, W W=V^{12 \beta-1-9 \beta / \alpha} Z^{(3-\alpha) / \alpha}\left[(\alpha-1)(\alpha-2) V^{-6 \beta}\right. \\
\left.+(3 \alpha-1)(3 \alpha-2) Z^{-2} f(Z)-\alpha(5 \alpha-3) Z^{-1} f^{\prime}(Z)+\alpha^{2} f^{\prime \prime}(Z)\right], \\
G, V V=V^{12 \beta-3-3 \beta / \alpha} Z^{(1-\alpha) / \alpha}\left[(3 \beta-1)(3 \beta-2) V^{-6 \beta}\right. \\
\left.+(3 \beta-1)(3 \beta-2) Z^{-2} f(Z)+9 \beta(3 \beta-1) Z^{-1} f^{\prime}(Z)+9 \beta^{2} f^{\prime \prime}(Z)\right] .
\end{gathered}
$$

From the first of these equations we see by inspection that $G_{, W W}=0$ precisely if either

$$
\alpha=1, \quad Z^{2} f^{\prime \prime}(Z)-2 Z f^{\prime}(Z)+2 f(Z)=0,
$$

or

$$
\alpha=2, \quad 2 Z^{2} f^{\prime \prime}(Z)-7 Z f^{\prime}(Z)+10 f(Z)=0 .
$$

In the first of these cases, (44), the two independent solutions are $f(Z)=Z$ and $f(Z)=Z^{2}$ leading to the pressure function

$$
p=-a e^{4 \beta^{3}}+b e^{2 \beta^{3}}=-a Z^{2}+b Z,
$$

where $a$ and $b$ are integration constants. Using the conservation equation (19) the energy density is then given by

$$
\rho=5 a Z^{2}-3 b Z
$$

The equation of state itself can be written in the form

$$
a(5 p+\rho)^{2}=2 b^{2}(3 p+\rho) .
$$

It can be shown that $p, \rho$ and $\mathrm{d} p / \mathrm{d} \rho$ cannot all be positive for this equation of state. It is therefore unphysical according to our criterion. We conclude the examination of this case by noting that the special cases $a=0$ and $b=0$ correspond to the equations of state $3 p+\rho=0$ and $5 p+\rho=0$ respectively. The first of these equations of state was already encountered above as an exactly solvable case while in the second case the Jacobi geometry (41) is flat.

Moving on to the case (45), two independent solutions are $f(Z)=Z^{2}$ and $f(Z)=Z^{5 / 2}$. The pressure and energy density can then be expressed as

$$
p=-a Z^{5 / 2}+b Z^{2}, \quad \rho=6 a Z^{5 / 2}-5 b Z^{2} .
$$

The equation of state can be written in the form

$$
a^{4}(6 p+\rho)^{5}=b^{5}(5 p+\rho)^{4} .
$$

Again this an unphysical equation of state for which $p, \rho$ and $\mathrm{d} p / \mathrm{d} \rho$ cannot all be zero simultaneously.

The second possibility for a null Killing tensor occurs if $G, V V=0$ which happens precisely if either

$$
\beta=\frac{1}{3}, \quad f^{\prime \prime}(Z)=0,
$$

or

$$
\beta=\frac{2}{3}, \quad 2 Z f^{\prime \prime}(Z)+3 f^{\prime}(Z)=0 .
$$

In the first of these cases, (51), we have $p=-a Z+b$ and $\rho=3 a Z-b$ leading to the equation of state

$$
3 p+\rho=2 b .
$$

The corresponding solution was found by Whittaker 23. Recall that the special case $b=0$ was shown in section 3 to admit a non-null Killing tensor. The corresponding Jacobi geometry therefore admits two inequivalent Killing tensors. Finally in the case given by (52) we recover Schwarzschild's interior solution with the equation of state parametrized as

$$
\begin{aligned}
& p=-a+b Z^{-1 / 2}, \\
& \rho=a .
\end{aligned}
$$

This concludes the discussion of possible null Killing tensor cases. 


\section{$5 \quad$ Non-null Killing tensor solutions}

To investigate the existence of non-null Killing tensors we must take the sum and the difference of the second derivatives $G, W W$ and $G, V V$ and set the resulting expression equal to zero according to the conditions (35). After multiplication by a suitable non-vanishing function we obtain

$$
\begin{array}{r}
Z^{(1-\alpha) / \alpha} V^{12 \beta-3-3 \beta / \alpha}(G, W W-\epsilon G, V V)=-\epsilon B(Z) V^{\nu_{1}}+Z^{2 / \alpha} A(Z) V^{\nu_{2}} \\
-\epsilon(3 \beta-1)(3 \beta-2) V^{\nu_{3}}+(\alpha-1)(\alpha-2) Z^{2 / \alpha} V^{\nu_{4}}=0,
\end{array}
$$

where we have introduced the notation

$$
\begin{array}{r}
A(Z)=\alpha^{2} f^{\prime \prime}(Z)-\alpha(5 \alpha-3) Z^{-1} f^{\prime}(Z)+(3 \alpha-1)(3 \alpha-2) Z^{-2} f(Z), \\
B(Z)=9 \beta^{2} f^{\prime \prime}(Z)+9 \beta(3 \beta-1) Z^{-1} f^{\prime}(Z)+(3 \beta-1)(3 \beta-2) Z^{-2} f(Z), \\
\left(\nu_{1}, \nu_{2}, \nu_{3}, \nu_{4}\right)=(0, \mu,-6 \beta, \mu-6 \beta),
\end{array}
$$

with $\mu=2-6 \beta / \alpha, \epsilon=1$ for Hamilton-Jacobi separation and $\epsilon=-1$ for complex decoupling. To analyze when the above expression vanishes identically we consider the dependence on the variable $V$. If the exponents $\nu_{i}(i=1,2,3,4)$ are all different then we see that the form of the coefficient of $V^{\nu_{4}}$ in (55) implies that either $\alpha=1$ or $\alpha=2$. In the first case we have $\nu_{2}=2(1-3 \beta)$ implying that $\beta \neq 1 / 3$. Inspection of the coefficient of $V^{\nu_{3}}$ then shows that we must have $\beta=2 / 3$ implying

$$
\left(\nu_{1}, \nu_{2}, \nu_{3}, \nu_{4}\right)=(0,-2,-4,-6) \text {. }
$$

Equating the remaining two coefficients to zero we obtain

$$
\begin{aligned}
& A(Z)=f^{\prime \prime}(Z)-2 Z^{-1} f^{\prime}(Z)+2 Z^{-2} f(Z)=0, \\
& B(Z)=4 f^{\prime \prime}(Z)+6 Z^{-1} f^{\prime}(Z)=0 .
\end{aligned}
$$

However, it's easy to see that this pair of equations is incompatible. Considering now the second case $\alpha=2$, we have

$$
\left.\left(\nu_{1}, \nu_{2}, \nu_{3}, \nu_{4}\right)=(0,2-3 \beta,-6 \beta, 2-9 \beta)\right) .
$$

Comparison with the coefficient of $V^{\nu_{3}}$ shows that $\beta=1 / 3$ leading to

$$
\left(\nu_{1}, \nu_{2}, \nu_{3}, \nu_{4}\right)=(0,1,-2,-1) \text {. }
$$

The conditions for the remaining coefficients then become

$$
\begin{aligned}
& A(Z)=4 f^{\prime \prime}(Z)-14 Z^{-1} f^{\prime}(Z)+20 Z^{-2} f(Z)=0, \\
& B(Z)=f^{\prime \prime}(Z)=0 .
\end{aligned}
$$

This system also turns out to be incompatible. We have thus shown that at least two of the exponents $\nu_{i}$ must be equal.

Suppose first that $\nu_{1}=\nu_{2}$, i.e., $\alpha=3 \beta$. Then it follows that

$$
\left.\left(\nu_{1}, \nu_{2}, \nu_{3}, \nu_{4}\right)=(0,0,-6 \beta,-6 \beta)\right),
$$

implying that there are now only two linearly independent functions of $V$ in (55), namely $V^{\nu_{1}}=1$ and $V^{\nu_{3}}=V^{-6 \beta}$. Setting the coefficient of the latter function equal to zero shows that one of the following two conditions must hold:

$$
\left\{\begin{array}{lll}
\alpha=1 & \beta=1 / 3, & \text { (I) } \\
\alpha=2 & \beta=2 / 3 . & \text { (II) }
\end{array}\right.
$$

As we shall see case (I) contains Buchdahl's $n=1$ polytrope solution 16 as a special case while case (II) corresponds to Buchdahl's $n=5$ polytrope solution 25] as recently generalized by Simon [3]. The corresponding equations of state will be discussed in the next section.

Going now to the remaining cases we note that $\nu_{1} \neq \nu_{3}$ always holds since $\beta \neq 0$. Consider therefore $\nu_{1}=\nu_{4}$ i.e. $\mu=6 \beta$ leading to

$$
\left(\nu_{1}, \nu_{2}, \nu_{3}, \nu_{4}\right)=(0,6 \beta,-6 \beta, 0) \text {. }
$$

There are now three linearly independent functions of $V$ in 55 . Inspecting the coefficient of one of those functions, $V^{\nu_{3}}$, we find that there are two possibilities, $\beta=1 / 3$ or $\beta=2 / 3$. The first of these values for $\beta$ is not compatible with $\mu=6 \beta$. The second value implies $\alpha=-2$. For the remaining two coefficients to vanish we then have the conditions

$$
\begin{aligned}
-\epsilon B(Z)+12 Z^{-1} & =-4 \epsilon f^{\prime \prime}(Z)-6 \epsilon Z^{-1} f^{\prime}(Z)+12 Z^{-1}=0, \\
A(Z) & =4 f^{\prime \prime}(Z)-26 Z^{-1} f(Z)+56 Z^{-2} f(Z)=0 .
\end{aligned}
$$


Again, this is an incompatible system.

The next case is $\nu_{2}=\nu_{3}$ corresponding to $\mu=-6 \beta$. We then have

$$
\left(\nu_{1}, \nu_{2}, \nu_{3}, \nu_{4}\right)=(0,-6 \beta,-6 \beta,-12 \beta) .
$$

There are again three linearly independent functions of $V$ in (55). In particular, from the coefficient of $V^{\nu_{4}}$ we find that either $\alpha=1$ or $\alpha=2$. The first of these values is incompatible with $\mu=-6 \beta$ so we consider now the second. The remaining equations to be satisfied in that case are

$$
\begin{gathered}
B(Z)=2\left[2 f^{\prime \prime}(Z)+9 Z^{-1} f^{\prime}(Z)+6 Z^{-2} f(Z)=0,\right. \\
Z A(Z)-12 \epsilon=4 Z f^{\prime \prime}(Z)-14 f^{\prime}(Z)+20 Z^{-1} f(Z)-12 \epsilon=0,
\end{gathered}
$$

Again it can easily be confirmed that this system is incompatible. Note finally that we always have $\nu_{3} \neq \nu_{4}$. We have therefore exhausted the possibilities to satisfy (55).

\section{Equations of state corresponding to non-null Killing tensors}

We now derive the equations of state corresponding to (I) and (II) of (63). The equation of state for case (I) will be discussed in some detail while we shall be content with just writing down the case (II) equation of state. In case (I) the condition (55) is reduced to

$$
-\epsilon B(Z)+Z^{2} A(Z)=0
$$

leading to the equation

$$
\left(Z^{2}-\epsilon\right) f^{\prime \prime}(Z)-2 Z f^{\prime}(Z)+2 f(Z)=0 .
$$

Two independent solutions of this equation are $f(Z)=Z$ and $f(Z)=Z^{2}+\epsilon$. It follows that we can formally reobtain the equation state (48) by letting $\epsilon \rightarrow 0$. However, as we shall see the physical properties of the equation of state is very much different when $\epsilon$ is nonzero. We write the general solution as

$$
f(Z)=a Z^{2}-2 b Z+a \epsilon,
$$

corresponding to the pressure function

$$
p=a e^{4 \nu}-2 b e^{2 \nu}+a \epsilon
$$

It follows that pressure and energy density can be parametrized as

$$
\begin{aligned}
& p=a Z^{2}-2 b Z+a \epsilon, \\
& \rho=-5 a Z^{2}+6 b Z-a \epsilon .
\end{aligned}
$$

The equation of state can be written explicitly in the form

$$
8 b^{2}(2 a \epsilon-3 p-\rho)=a(4 a \epsilon-5 p-\rho)^{2} .
$$

Setting $a=0$ we again have the equation of state $3 p+\rho=0$. Setting $b=0$ gives the equation of state $5 p+\rho=4 a \epsilon$. It is convenient to write the equation of state as

$$
\begin{aligned}
& p=a\left(Z^{2}-2 \delta Z+\epsilon\right), \\
& \rho=a\left(-5 Z^{2}+6 \delta Z-\epsilon\right),
\end{aligned}
$$

where $\delta=b / a$. The parameter $a$ is then seen to be a scaling which does not matter for physical quantities such as $\mathrm{d} p / \mathrm{d} \rho$ or the relativistic adiabatic index $\gamma=(1+\rho / p)(\mathrm{d} p / \mathrm{d} \rho)$. Its absolute value is also irrelevant for inequalities such as $p>0$ and energy conditions such as $\rho>3 p$. However, its sign does matter and must be determined in each case. For example, suppose the equation of state is given by $p=a x^{2}, \rho=a x$ corresponding to $p=a^{-1} \rho^{2}$. For $a>0$ this is a $n=1$ polytrope while for $a<0$ it is clearly unphysical. Moreover, given any solution of the Einstein equations $g_{\alpha \beta}$ for any equation of state $p=\Phi(x), \rho=\Psi(x)$, the scaling properties of the Einstein equations guarantee that the scaled metric $a^{-1} g_{\alpha \beta}$ together with the scaled equation of state $p=a \Phi(x), \rho=a \Psi(x)$ also solve the Einstein equations. In fact, this is the relativistic version of a mechanical similarity transformation.

For the Hamilton-Jacobi case $(\epsilon=1)$ with $\delta=1$ we recover Buchdahl's $n=1$ polytrope solution [16]. The harmonic solution and the Hamilton-Jacobi solution with $\delta \neq 1$ have apparently not appeared in the literature before. We now take a first look at the physial properties of these equations of state. The velocity of sound is given by the formula

$$
v_{\text {sound }}^{2}=\frac{\mathrm{d} p}{\mathrm{~d} \rho}=\frac{\delta-Z}{5 Z-3 \delta} .
$$


Since $Z>0$ by definition the condition $0 \leq \mathrm{d} p / \mathrm{d} \rho \leq 1$ can only be satisfied if $\delta>0$ and

$$
\frac{2 \delta}{3} \leq Z \leq \delta
$$

The energy condition

$$
\rho+p=4 a Z(-Z+\delta) \geq 0
$$

then shows that we must have $a>0$. Considering now the condition $p \geq 0$ we first note that if $\epsilon=-1$, then that condition is nowhere satisfied in the region given by 76 . Thus the harmonic solution is nowhere physical according to the adopted criterion and we shall not consider it further.

For the Hamilton-Jacobi solution, $\epsilon=1$, we find that if $\delta \leq 1$ then $p \geq 0$ automatically while if $\delta>1$ we have the further condition

$$
Z \leq \delta-\sqrt{\delta^{2}-1}
$$

Compatibility of $\left(78\right.$ ) with $(76)$ now leads to a restriction on $\delta$ given by $\delta / 3>\sqrt{\delta^{2}-1}$. Since $\delta>0$ this is equivalent to

$$
\delta<\frac{3 \sqrt{2}}{4} \approx 1.06
$$

Summarizing our analysis so far, the conditions $p \geq 0$ and $0 \leq \mathrm{d} p / \mathrm{d} \rho \geq 1$ are both satisfied if $\epsilon=1$ and the conditions

$$
0<\delta<\frac{3 \sqrt{2}}{4}, \quad \frac{2 \delta}{3} \leq Z \leq f(\delta),
$$

are satisfied and where $f(\delta)$ is defined by

$$
f(\delta)= \begin{cases}\delta & \text { for } \delta \leq 1 \\ \delta-\sqrt{\delta^{2}-1} & \text { for } \delta>1\end{cases}
$$

Examining now the condition $\rho \geq 0$, we find that the polynomial $-5 Z^{2}+6 \delta Z-1$ must have two distinct real roots (excluding the $Z=$ constant case). It follows that $\delta>\sqrt{5} / 3 \approx 0.745$. Combining the condition $\rho \geq 0$ with (80) gives the final result that the equation of state is physical according to our criterion in the range

$$
\frac{2 \delta}{3} \leq Z \leq g(\delta)
$$

where

$$
g(\delta)= \begin{cases}\left(3 \delta+\sqrt{9 \delta^{2}-5}\right) / 5 & \text { for } \delta \leq 1 \\ \delta-\sqrt{\delta^{2}-1} & \text { for } \delta>1\end{cases}
$$

The requirement $2 \delta / 3<g(\delta)$ further restricts the parameter values to the range

$$
\frac{3}{4}<\delta<\frac{3 \sqrt{2}}{4}
$$

Furthermore the limit $p \rightarrow 0$ can only be reached if $\delta \geq 1$. Therefore, if $\delta<1$, this equation of state cannot be used all the way out to the star surface. One way comparing the equation of state with $\delta \neq 1$ with Buchdahl's original equation of state is to compute the ratio $p_{*} / \rho_{*}$ between the pressure and energy density in the limit $v_{\text {sound }} \rightarrow 1$. The result is

$$
\frac{p_{*}}{\rho_{*}}=\frac{9-8 \delta^{2}}{16 \delta^{2}-9} .
$$

This shows that the ratio is smaller for $\delta>1$ and bigger for $\delta<1$. A larger ratio can be interpreted as corresponding to a stiffer equation of state. Another way of examining the physical nature of the equation of state is to calculate the relativistic adiabatic index

$$
\gamma=\frac{p+\rho}{p} \frac{\mathrm{d} p}{\mathrm{~d} \rho}
$$

It can be shown that $\gamma$ increases with $\delta$. This is also an indication that equations of state with larger $\delta$ are stiffer or less compressible.

We turn now to case (II) of 63 . The pressure function is given by

$$
p=f(Z)=a F_{1}(Z)+b F_{2}(Z)
$$

where

$$
\begin{aligned}
& F_{1}(Z)=Z^{2}+\frac{10}{3} \epsilon Z+1 \\
& F_{2}(Z)=Z^{-1 / 2}\left(Z^{3}+15 \epsilon Z^{2}+15 Z+\epsilon\right) .
\end{aligned}
$$


Using the variable $Y=Z^{1 / 2}=e^{\nu}$ the equation of state for the Hamilton-Jacobi case $(\epsilon=1)$ can be written in parametric form as (following Simon [3])

$$
\begin{aligned}
& p=(6 Y)^{-1}\left[-\rho_{+}(1+Y)^{6}+\rho_{-}(1-Y)^{6}\right], \\
& \rho=\rho_{+}(1+Y)^{5}+\rho_{-}(1-Y)^{5},
\end{aligned}
$$

where

$$
\begin{aligned}
& \rho_{+}=-\frac{1}{2}(a+6 b), \\
& \rho_{-}=-\frac{1}{2}(a-6 b) .
\end{aligned}
$$

The subcase $\rho_{+}=0$ is Buchdahl's polytrope of index $n=5$ [25]. The general case $\rho_{+} \neq 0$ was recently discussed by Simon [3].

The equation of state for the harmonic case, $\epsilon=-1$, can be written as

$$
p=(6 Y)^{-1} \Re e\left[\rho_{0}(1+i Y)^{6}\right], \quad \rho=\Im m\left[\rho_{0}(1+i Y)^{5}\right],
$$

where $\rho_{0}$ is a complex constant. We have not found any region where this equation of state is physical.

\section{Solving the configuration equations}

In this section we outline the procedure for integrating the Hamiltonian configuration equations for equations of state which correspond to a Jacobi geometry which admits a second rank Killing tensor. We begin by expressing the Hamiltonian (30) in terms of the symmetry adapted variables defined by the ansatz (40)

$$
H=-\frac{1}{2} W_{, R} V_{, R}+24 \alpha \beta\left[W^{\alpha-1} V^{3 \beta-1}+\kappa W^{3 \alpha-1} V^{3 \beta-1} f(Z)\right],
$$

where the relative radial gauge is given by

$$
\mathcal{N}=\alpha \beta(W V)^{-1}=12 \alpha \beta W^{-(\alpha+3 \beta) /(3 \beta)} Z^{-1 /(3 \beta)},
$$

while the absolute radial gauge becomes

$$
N=N_{T} \mathcal{N}=12 \alpha \beta W^{(3 \alpha-2) / 2} V^{(3 \beta-2) / 2} .
$$

We are interested in how the elementary flatness condition (21) restricts the integration constants as we perform the integration. For that purpose we need to express the Schwarzschild radial gauge (5) in the symmetry adapted variables

$$
\begin{aligned}
N_{\mathrm{S}}{ }^{-1} & =\alpha N^{-1} W^{\alpha-1} W_{, R}=\frac{1}{12} \beta^{-1} W^{-\alpha / 2} V^{(2-3 \beta) / 2} W_{, R} \\
& =\frac{1}{12} \beta^{-1} W^{\alpha(1-3 \beta) /(3 \beta)} Z^{(2-3 \beta) /(6 \beta)} W_{, R} .
\end{aligned}
$$

In the symmetry adapted variables the Hamiltonian 92 has the form

$$
H=-\frac{1}{2} W_{, R} V_{R}+A(W) V+B(W) .
$$

The procedure to obtain the general solution for this type of Hamiltonian has been outlined in [17]. The fact that the potential in $(96)$ is linear in one of the null variables, $(V)$, leads to a decoupled equation of motion for the other null variable, $(W)$,

This equation has the first integral

$$
W, R R=2 A(W)
$$

$$
E=\frac{1}{2}\left(W_{, R}^{2}\right)-2 \int A(W) \mathrm{d} W .
$$

By integrating this equation we can express $W$ as a function of $R$. Inserting this into the Hamiltonian constraint results in the linear first order equation for $V$ given by

$$
V_{, R}=(2 / W, R)[A(W) V+B(W)] .
$$

The solution of this equation is

$$
V=2 W_{, R} \int B\left(W_{, R}\right)^{-2} \mathrm{~d} R .
$$

Rather than deriving the metrics for all the possible models we shall give a few examples which illustrate the technique. First of all we show how to calculate the metrics for Schwarzschild's exterior and interior solutions in the Hamiltonian framework. After that we calculate the metric for the generalization of Buchdahl's $n=1$ polytrope solution. In the final subsection we discuss how the remaining models can be integrated. The equations of state which correspond to minisuperspace Killing tensors are summarized in table 8 . 
Table 2: Perfect fluids corresponding to Killing tensor solutions. In the first two columns a "-" means that the value of $\alpha$ or $\beta$ is arbitrary. The parameters $a, b, \rho_{ \pm}$are real constants while $\rho_{0}$ is a complex constant. The notations $Y=e^{\nu}$ and $Y_{ \pm}=1 \pm Y$ are also used. For the case $\alpha=2, \beta=2 / 3$, it is not possible to write down an equation of state in closed form. Instead the expressions for $\rho(Y)$ are given in the fourth column. The notation "H-J" in the last column stands for a Hamilton-Jacobi Killing tensor type. The Hamilton-Jacobi case with $(\alpha, \beta)=(1,1 / 3)$ reduces to a Killing vector solution when $a=b$.

\begin{tabular}{lllll}
\hline$\alpha$ & $\beta$ & $p=p(Y)$ & $\begin{array}{c}\text { equation of state } \\
{[\rho=\rho(Y) \text { in the last two rows }]}\end{array}$ & Killing tensor type \\
\hline 1 & - & $-a Y^{4}+b Y^{2}$ & $a(5 p+\rho)^{2}=2 b^{2}(3 p+\rho)$ & null \\
2 & - & $-a Y^{5}+b Y^{4}$ & $a^{4}(6 p+\rho)^{5}=b^{5}(5 p+\rho)^{4}$ & null \\
- & $1 / 3$ & $-a Y^{2}+b$ & $3 p+\rho=2 b$ & null \\
- & $2 / 3$ & $-a+b Y^{-1}$ & $\rho=a$ & null \\
1 & $1 / 3$ & $a Y^{4}-2 b Y^{2}+a$ & $\frac{(5 p+\rho-4 a)^{2}}{2 a-3 p-\rho}=\frac{8 b^{2}}{a}$ & H-J \\
1 & $1 / 3$ & $a Y^{4}-2 b Y^{2}-a$ & $\frac{(5 p+\rho+4 a)^{2}}{2 a+3 p+\rho}=-\frac{8 b^{2}}{a}$ & harmonic \\
2 & $2 / 3$ & $(6 Y)^{-1}\left(-\rho_{+} Y_{+}^{6}+\rho_{-} Y_{-}^{6}\right)$ & $\rho_{+} Y_{+}^{5}+\rho_{-} Y_{-}^{5}$ & H-J \\
2 & $2 / 3$ & $(6 Y)^{-1} \Re e\left[\rho_{0}(1+i Y)^{6}\right]$ & $\Im m\left[\rho_{0}(1+i Y)^{5}\right]$ & harmonic \\
\hline
\end{tabular}

\subsection{Schwarzschild's exterior solution}

To illustrate how the Hamiltonian method works for the static models we first derive the Schwarzschild vacuum solution. In this case there is no matter term and choosing $\alpha=1, \beta=1 / 3$, the Hamiltonian (92) becomes

$$
H=-\frac{1}{2} W,_{R} V_{, R}+8 \text {. }
$$

The problem is now trivially solvable. In fact the Jacobi geometry is obviously flat in this case. The Hamiltonian constraint gives $W, R=C$ and $V_{, R}=16 / C$ where $C$ is a separation constant. Since $r=e^{\beta^{1}}=W$ it follows that $R=$ $C^{-1} r$ (up to a gauge translation) and $V=16 C^{-1}\left(R-R_{0}\right)$. The radial gauge is given by $N=N_{T} \mathcal{N}=4 W^{1 / 2} V^{-1 / 2}$. Using the relations to the metric variables, $e^{2 \beta^{3}}=W^{-1} V, N^{2}=16 W V^{-1}$, one then arrives at the usual form of the Schwarzschild metric with the mass given by $M=C R_{0} / 2$.

\subsection{Schwarzschild's interior solution, $\rho=\rho_{\mathrm{s}}$}

The equation of state is parametrized according to (54) as $p=f(Z)=-a+b Z^{-1 / 2}$ and $\rho=\rho_{\mathrm{s}}=a$. Further we have $\beta=2 / 3$ and we set $\alpha=1$ implying $W=r$ and $Z=W^{-1} V^{2}$. Referring to (96) we also have

$$
A(W)=16\left(1-\kappa a W^{2}\right), \quad B(W)=16 \kappa b W^{5 / 2} .
$$

Applying the procedure outlined above we have the following first integral

$$
E=\frac{1}{2} W_{, R}^{2}-32\left(W-\frac{1}{3} \kappa a W^{3}\right) .
$$

The next step is to use the condition of elementary flatness 21) to determine the value of $E$. It follows form (95) that $W, R$ can be expressed in terms of the Schwarzschild radial gauge as

$$
W, R=8 W^{1 / 2} N_{\mathrm{S}}{ }^{-1} .
$$

Elementary flatness requires that $N_{\mathrm{S}} \rightarrow 1$ as $r=W \rightarrow 0$. This implies that $W_{, R}=0$ at the center of the star. It then follows that $E=0$ for regular models. The equation $(103)$ can then be integrated further in terms of elementary functions. Alternatively one can use an intrinsic variable 17] such as $W$ or a function of $W$ as coordinate in place of $R$. In fact, it is the intrinsice variable choice $W=r$ which gives us the usual form of the metric. In practice, what 
we must do to use an intrinsic variable is to change the integration variable in (100). Using $W$ as the new integration variable that equation reads

$$
V=2 W, R \int B(W)(W, R)^{-3} \mathrm{~d} W
$$

Performing the integration gives

$$
V=\frac{1}{2} a W^{1 / 2}\left(3 b^{-1}-\kappa K \sqrt{1-\frac{1}{3} \kappa a W^{2}}\right),
$$

where $K$ is an integration constant. Using $Z=W^{-1} V^{2}$ gives one of the metric coefficients

$$
Z=e^{2 \nu}=\frac{1}{4} b^{2} \kappa K\left(3 D-\sqrt{1-\frac{1}{3} \kappa \rho_{\mathrm{s}} r^{2}}\right)^{2},
$$

where $D=(\kappa a K)^{-1}$. Finally, using 104 to compute the remaining metric coefficient yields

$$
e^{2 \lambda}=N_{\mathrm{S}}^{2}=64 W(W, R)^{-2}=\left(1-\frac{1}{3} \kappa \rho_{\mathrm{s}} r^{2}\right)^{-1} .
$$

Using (25) we also obtain the mass from this expression as $M=\kappa \rho_{\mathrm{s}} r_{\mathrm{s}}{ }^{3}$. The physical meaning of the constant $D$ comes from evaluating (107) at the surface. This gives $D=\left(1-\frac{1}{3} \kappa \rho_{\mathrm{s}} r_{\mathrm{s}}{ }^{2}\right)^{1 / 2}$ where we have used $Z_{\mathrm{s}}=(b / a)^{2}$. These relations together with (107) and (108) give the standard form of Schwarzschild's interior metric as given in [14]. The constant $b$ corresponds to a gauge scaling of the time coordinate and consequently has no physical meaning.

\subsection{Buchdahl's polytrope of index one and its generalization}

In this section we calculate the metric components for the solution corresponding to case (I) of (63). This case generalizes Buchdahl's $n=1$ polytropic solution to a fluid which is no longer gas-like in the limit $p=0$ but rather has a non-zero density there, $\rho_{\mathrm{s}} \neq 0$. Since $\alpha=1$ and $\beta=1 / 3$ we have $Z=V / W$ and the symmetry adapted null variables are $W=e^{w}$ and $V=e^{3 v}$. Specializing the Hamiltonian (92) to this case yields

$$
H=-\frac{1}{2} W_{, R} V_{R}+8\left[1+\kappa a\left(V^{2}-2 \delta W V+W^{2}\right)\right] .
$$

Separation is achieved by introducing the symmetry adapted non-null variables $(T, X)$ by

$$
\begin{aligned}
& T=\frac{1}{2}(W+V)=\frac{1}{2} e^{\beta^{1}}\left(1+e^{2 \beta^{3}}\right)=\frac{1}{2} r(1+Z), \\
& X=\frac{1}{2}(W-V)=\frac{1}{2} e^{\beta^{1}}\left(1-e^{2 \beta^{3}}\right)=\frac{1}{2} r(1-Z) .
\end{aligned}
$$

In terms of these variables the Hamiltonian takes the manifestly separable form

$$
H=\frac{1}{2}\left(-T_{, R}^{2}+X_{, R}^{2}\right)+8\left[1-2 \kappa a(\delta-1) T^{2}+2 \kappa a(\delta+1) X^{2}\right],
$$

leading to the separated equations

$$
\begin{aligned}
T_{, R}{ }^{2}+32 \kappa a(\delta-1) T^{2} & =K+16, \\
X_{, R}{ }^{2}+32 \kappa a(\delta+1) X^{2} & =K,
\end{aligned}
$$

where $K$ is the separation constant. It is obvious from the second of these equations that $K>0$. It is also clear that the solutions $T(R)$ will have different functional forms for the three cases $\delta<1, \delta=1$ and $\delta>1$. Since $T$ is a cyclic variable in the Hamiltonian (111) when $\delta=1$ it follows that the original Buchdahl $n=1$ polytrope solution corresponds to the existence of a Killing vector in the minisuperspace Jacobi geometry.

As explained in section 5 , when $\delta<1$ the equation of state has no zero pressure limit. Although such an equation of state could still be useful for high pressures we restrict the following discussion to the cases with $\delta \geq 1$. The solutions are given by

$$
\begin{aligned}
T(R) & = \begin{cases}4(\cosh \zeta)\left(R-R_{-}\right), & \text {for } \delta=1 \\
4(\cosh \zeta) \omega_{-}^{-1} \sin \left[\omega_{-}\left(R-R_{-}\right)\right], & \text {for } \delta>1\end{cases} \\
X(R) & =4(\sinh \zeta) \omega_{+}{ }^{-1} \sin \left[\omega_{+}\left(R-R_{+}\right)\right],
\end{aligned}
$$

where $\zeta=\operatorname{arcsinh}(\sqrt{K} / 4), \omega_{ \pm}=\sqrt{32 a \kappa(\delta \pm 1)}$, and the parameters $R_{ \pm}$are integration constants. Let us now consider the possible range of values which $T$ and $X$ can assume. It is obvious from (110) that $T \geq 0$. Also, it follows from (32) that $Z \leq 1$ throughout the interior of the star implying that $X \geq 0$. It is also clear from (110) that both $T$ and $X$ vanish at the center. Next we use the translational freedom in the radial coordinate to make it vanish at 
the center, $R_{\mathrm{c}}=0$. The condition $T(0)=0$ then implies $R_{-}=0$ for $\delta=1$ and that $\omega_{-} R_{-}$is a multiple of $\pi$ when $\delta>1$. Further $X(0)=0$ implies that $\omega_{+} R_{+}$must be a multiple of $\pi$. Since $\alpha \beta=1 / 3>0$ it is clear from (94) that the radial gauge function is positive and hence $R$ increases outward from the center. To ensure the positivity of $T$ and $X$ when $R>0$ it follows that $\omega_{+} R_{+}$and $\omega_{-} R_{-}$must both be even multiples of $\pi$. But then we can just as well set $R_{ \pm}=0$ without loss of generality.

We now consider the elementary flatness condition (21) at the center of the stellar model. In this case the condition can be transformed to

$$
\lim _{R \rightarrow 0} \frac{1}{4} W_{, R} Z^{1 / 2}=1 .
$$

For the above solution we have $\left(T_{, R}\right)_{\mathrm{c}}=4 \cosh \zeta,\left(X_{, R}\right)_{\mathrm{c}}=4 \sinh \zeta$ leading to $\left(W,_{R}\right)_{\mathrm{c}}=4 e^{\zeta}$ and $(V, R)_{\mathrm{c}}=4 e^{-\zeta}$. The central value of $Z$ is given by the indeterminate expression $Z_{\mathrm{c}}=(V / W)_{\mathrm{c}}$ which by the previous argument is seen to be given by $Z_{\mathrm{c}}=e^{-2 \zeta}$. Using these central values we find that the elementary flatness condition is already satisfied without restrictions on the remaining integration constant $\zeta$. Restricting attention now to the case $\delta>1$, the final form of the solution is therefore given by

$$
\begin{aligned}
& T(R)=4(\cosh \zeta)\left(\omega_{-}\right)^{-1} \sin \left(\omega_{-} R\right), \\
& X(R)=4(\sinh \zeta)\left(\omega_{+}\right)^{-1} \sin \left(\omega_{+} R\right) .
\end{aligned}
$$

Referring to $(76)$ and $(78)$ we must require for consistency that $2 \delta / 3<Z_{\mathrm{c}}<Z_{\mathrm{s}}$ leading to a restriction on the separation parameter

$$
2 \delta / 3<e^{-2 \zeta}<\delta-\sqrt{\delta^{2}-1} .
$$

The central values of the pressure and energy density can be inferred from (74) as functions of $\delta$ and $\zeta$. Using (94) to calculate the radial gauge we find that the metric for this model is given by

$$
\mathrm{d} s^{2}=-Z \mathrm{~d} t^{2}+16 Z^{-1} \mathrm{~d} R^{2}+W^{2} \mathrm{~d} \Omega^{2},
$$

where $Z=V / W=(T-X) /(T+X), W=T+X$ and $T$ and $X$ are given by (115). The physical properties of this model will be given elsewhere.

\subsection{Other models}

We begin this subsection by discussing how the remaining null Killing tensor models can be integrated. Whittaker's solution with equation of state given by (53) has a structure which is similar to Schwarzschild's interior solution. The Hamiltonian is again linear in $V$ and thus the solution can be integrated in an analogous manner. In this case the condition for elementary flatness does not restrict the integration constant $E$ to be zero but the resulting equations can nevertheless be integrated in terms of elementary functions in the Schwarzschild radial gauge. This leads back to the form for the solution given by Whittaker 23. For the null Killing tensor cases corresponding to the equations of state (48) and (50) the roles of $W$ and $V$ are reversed. This gives a Hamiltonian which is linear in $W$ leading to a decoupled equation in $V$. Although the integration proceeds in an analogous way it is less straightforward to impose the elementary flatness condition. This is because that condition is expressed in terms $W$. However, an argument can be made using the Hamiltonian constraint to express the flatness condition in terms of $V$. These solutions cannot in general be expressed in Schwarzschild coordinates.

The second Hamilton-Jacobi solution corresponding to the equation of state (89) can be integrated in much the same way as Buchdahl's $n=1$ polytrope and its generalization were treated in section 7.3 . Buchdahl's equation of state corresponding to $\rho_{+}=0$ tends to a Newtonian $n=5$ polytrope in the zero pressure limit. In fact, like the Newtonian $n=5$ polytrope, the star has an inifinite radius. However, the more general Simon solutions do not seem to have this property and a closer examination of the physical properties of this class of solutions would be of interest.

Although the harmonic solutions found in this work do not seem to of physical interest we briefly indicate the method by which they can be integrated. The decoupling variables in these cases are complex and given by $Q=W+i V$ and its complex conjugate $\bar{Q}=W-i V$. Using these variables together with the pressure functions (74) (with $\epsilon=1$ ) and (91) the Hamiltonian (92) becomes explicitly separated into a $Q$-dependent and a $\bar{Q}$-dependent part respectively. The procedure to obtain the metric variables then parallels that for the Hamilton-Jacobi case.

\section{Concluding remarks}

We have shown that a number of known exact solutions for static star configurations can be described in a unified setting using an ADM-like minisuperspace geometrization of the dynamics. New solutions have also been found in this process illustrating the power of the Hamiltonian formalism. The remark by Kramer et al. in 1980 (14], p.130-131) 
that "A Hamiltonian formulation ... is not well-adapted to searching for exact solutions" reflected a prejudice of the time which should be dispelled by now. Indeed, the traditional view towards exact solutions is that, as stated by Schutz in 1985 when discussing Buchdahl's $n=1$ polytropic solution, that "Finding such [exact] solutions is an art which requires the successful combination of useful coordinates, simple geometry, good intuition, and in most cases luck" (26], p.263). In the light of the present work as well as earlier work on spatially homogeneous models (see [17] and references therein), the opposite view seems perhaps more appropriate. At least those exact solutions which correspond to symmetries and also many submanifold solutions 27 can certainly be derived by systematic methods.

\section{References}

[1] K. Schwarzschild, Sitz. Preuss. Akad. Wiss. 424 (1916).

[2] L. Lindblom and A. K. M. Masood-ul-Alam, in Directions in General Relativity II: Papers in Honor of Dieter Brill, edited by B. L. Hu and T. A. Jacobson (Cambridge University Press, Cambridge, 1993), p. 172.

[3] W. Simon, Gen. Rel. Grav. 26, 97 (1994).

[4] C. Uggla, K. Rosquist, and R. T. Jantzen, Phys. Rev. D 42, 404 (1990).

[5] K. Rosquist and C. Uggla, J. Math. Phys. 32, 3412 (1991).

[6] C. Uggla, R. T. Jantzen, K. Rosquist, and H. von Zur-Mühlen, Gen. Rel. Grav. 23, 947 (1991).

[7] A. Ashtekar, R. Tate, and C. Uggla, Int. J. Mod. Phys. D 2, 15 (1993).

[8] K. Rosquist and C. Uggla, Mod. Phys. Lett. A 8, 2815 (1993).

[9] C. W. Misner, K. S. Thorne, and J. A. Wheeler, Gravitation (Freeman, San Fransisco, USA, 1973).

[10] K. Rosquist, C. Uggla, and R. T. Jantzen, Class. Quantum Grav. 7, 611 (1990).

[11] K. Rosquist, C. Uggla, and R. T. Jantzen, Class. Quantum Grav. 7, 625 (1990).

[12] R. C. Tolman, Phys. Rev. 55, 364 (1939).

[13] H. Knutsen, Gen. Rel. Grav. 22, 925 (1990).

[14] D. Kramer, H. Stephani, M. A. H. MacCallum, and E. Herlt, Exact Solutions of the Einstein Equations (VEB Deutscher Verlag der Wissenschaften, Berlin, GDR, 1980).

[15] K. Rosquist, J. Math. Phys. 30, 2319 (1989).

[16] H. A. Buchdahl, Astrophys. J. 147, 310 (1967).

[17] C. Uggla, R. T. Jantzen, and K. Rosquist, in preparation (unpublished).

[18] B. K. Harrison, K. S. Thorne, M. Wakano, and J. A. Wheeler, Gravitation theory and gravitational collapse (University of Chicago Press, Chicago, USA, 1965).

[19] A. H. Taub, Ann. Math. 53, 472 (1951).

[20] R. T. Jantzen, Phys. Rev. D 37, 3472 (1988).

[21] A. Ashtekar, Lectures on non-perturbative canonical gravity (World Scientific, Singapore, 1991).

[22] M. Goliath and K. Rosquist, Report, Department of Physics, Stockholm University, (unpublished), in preparation.

[23] J. M. Whittaker, Proc. Roy. Soc. Lond. A 306, 1 (1968).

[24] R. F. Tooper, Astrophys. J. 142, 1541 (1965).

[25] H. A. Buchdahl, Astrophys. J. 140, 1512 (1964).

[26] B. F. Schutz, A first course in general relativity (Cambridge University Press, Cambridge, U.K., 1985).

[27] R. T. Jantzen, C. Uggla, and K. Rosquist, Gen. Rel. Grav. 25, 409 (1993). 